\title{
Inspiratory muscle strength in subjects with tetraplegia: viability of evaluation through the measurement of maximal inspiratory pressure
}

\author{
Força muscular inspiratória em sujeitos com tetraplegia: viabilidade \\ da avaliação pela medida da pressão inspiratória máxima
}

\author{
Marlene Aparecida Moreno ${ }^{[a]}$, Juliana Viana Paris ${ }^{[b]}$, Raphael do Nascimento Pereira ${ }^{[c]}$, \\ Antonio Roberto Zamuné ${ }^{[\mathrm{d}]}$, Tais Mendes de Camargo ${ }^{[\mathrm{e}]}$, Ricardo Machado Leite de Barros ${ }^{[\mathrm{f}]}$ \\ [a] PhD, professor, Universidade Metodista de Piracicaba, Faculdade de Ciências da Saúde, Piracicaba, SP - Brazil, e-mail: \\ ma.moreno@terra.com.br \\ [b] MSc, Universidade Estadual de Campinas, Campinas, SP - Brazil, e-mail: julianav.paris@gmail.com \\ [c] PhD candidate, Universidade Metodista de Piracicaba, Piracicaba, SP - Brazil, e-mail: raphaelnpfisio@yahoo.com.br \\ [d] PhD candidate, Universidade Federal de São Carlos, São Carlos, SP - Brazil, e-mail: beto.zam@gmail.com \\ [e] PhD candidate, Universidade Metodista de Piracicaba, Piracicaba, SP - Brazil, e-mail: taismendesdecamargo@gmail.com \\ [f] PhD, professor, Universidade Estadual de Campinas, Campinas, SP - Brazil, e-mail: ricardomachado12@yahoo.com.br
}

\begin{abstract}
Objective: To analyze the values of maximal inspiratory pressure (MIP) and sniff nasal inspiratory pressure (SNIP) and to verify the existence of concordance between the two evaluation methodologies, in subjects with tetraplegia. Materials and methods: Cross-sectional study with 17 tetraplegic men, aged 30.42 \pm 7.67 years, who underwent MIP and SNIP evaluation using a respiratory pressure meter. Results: The MIP and SNIP values obtained showed no difference when compared to each other $88.42 \pm 29.39$ vs. 86.68 $\pm 25.40 \mathrm{cmH}_{2} \mathrm{O}$, respectively). They were, however, significantly lower compared to the predicted values $\left(\mathrm{MIP}=128.92 \pm 7.18\right.$; SNIP $\left.=114.11 \pm 3.19 \mathrm{cmH}_{2} \mathrm{O}\right)$, with the MIP values presenting correlation $\left(\mathrm{r}^{2}=0.94\right.$; $\mathrm{p}<0.0001$ ) and concordance with those of the SNIP. Conclusions: Both the MIP and SNIP values obtained were lower than the predicted values, indicating a reduction in inspiratory muscle strength (IMS). Both
\end{abstract}


techniques showed correlation and concordance, suggesting that MIP can be used as a noninvasive method for IMS evaluation in this population.

Keywords: Spinal cord diseases. Respiratory function tests. Respiratory muscles. Muscle strength.

\section{Resumo}

Objetivo: Analisar os valores da pressão inspiratória máxima ( $\left.P I_{\text {máx }}\right)$ e pressão inspiratória nasal sniff (Pnsn), bem como verificar a existência de concordância entre as duas metodologias de avaliação, em sujeitos com tetraplegia. Materiais e métodos: Estudo transversal com 17 homens tetraplégicos, idade 30,42 \pm 7,67 anos, os quais foram submetidos a avaliação da PI máx e da Pnsn, por intermédio da manovacuometria. Resultados: Os valores obtidos da PI $I_{\text {máx }}$ e da Pnsn não apresentaram diferença quando comparados entre si (88,42 $\pm 29,39$ vs. $86,68 \pm 25,40 \mathrm{cmH}_{2} \mathrm{O}$, respectivamente), no entanto, foram significativamente menores em relação aos valores preditos $\left(\mathrm{PI}_{\text {máx }}=128,92 \pm 7,18 ;\right.$ Pnsn $\left.=114,11 \pm 3,19 \mathrm{cmH}_{2} \mathrm{O}\right)$, tendo os valores da $\mathrm{PI}_{\text {máx }}$ apresentado correlação $\left(r^{2}=0,94 ; p<0,0001\right)$ e concordância com os da Pnsn. Conclusões: Tanto os valores obtidos pela PI máx quanto pela Pnsn mostraram-se inferiores aos preditos, indicando redução da força muscular inspiratória (FMI). As duas técnicas apresentaram correlação e concordância, sugerindo que a PI máx pode ser utilizada como um método não invasivo para avaliação da FMI nesta população.

Palavras-chave: Doenças da medula espinhal. Testes de função respiratória. Músculos respiratórios.

Força muscular.

\section{Introduction}

Subjects with spinal cord injury present serious alterations in respiratory function due to sensory and motor impairment below the level of the injury, these alterations are characterized by paralysis or respiratory muscle weakness $(1,2)$. This impairment can be evidenced by the considerable decrease in respiratory muscle strength, evaluated by measuring the maximal respiratory pressures $(3,4)$. However, in people with spinal cord injury, the values obtained from these measurements are questioned, as they are too variable to be considered as an index of inspiratory muscle strength (IMS) (5).

Alterations in ventilatory mechanics and in IMS may hinder the performance of this maneuver and thus promote inadequate results $(6,7)$. Since these are volitional tests, the measures depend on the comprehension and cooperation of the subject to perform maximal voluntary efforts $(8,9)$, and thus, underestimated values are set when faults occur in the comprehension, motivation, and coordination, or when the patient presents fatigue or discomfort while performing the maneuvers (7). Aiming to minimize the difficulties encountered in the measurement of IMS from the measurement of maximal inspiratory pressure (MIP), new methods of non-invasive evaluation have been proposed, with the sniff nasal inspiratory pressure (SNIP) test being one of these, which correlates with esophageal pressure, considered the gold standard for evaluating IMS, in healthy individuals and patients with musculoskeletal and neuromuscular diseases $(7,10)$. In healthy subjects, SNIP can be used as the maneuver of choice for evaluating IMS, as it reproduces the predicted values (11). Thus, it was hypothesized that from the evaluation of SNIP it would be possible to verify the viability of IMS measurement in tetraplegic people through MIP measurement, as, in the presence of low MIP values, SNIP offers a way to differentiate inspiratory muscle weakness from the difficulty in achieving sustained continual effort (7).

Considering that the functional evaluation of the inspiratory muscles is important for determining the degree of dysfunction of these muscles in people with spinal cord injury, and given the need to clarify the viability of using MIP for the evaluation of IMS in this population, this study aimed to evaluate MIP and SNIP and to verify the existence of correlation and concordance between the values obtained from the two methodologies in a sample of subjects with tetraplegia. 


\section{Materials and methods}

Design

Cross-sectional study.

\section{Ethical considerations}

The present study was approved by the human research ethics committee of the Institution, under protocol No. 55/08. The volunteers that agreed to participate signed the Terms of Free Prior Informed Consent, according to the criteria of Resolution 196/96 of the National Health Council.

\section{Study group and inclusion criteria}

The sample was composed of 17 quadriplegic male volunteers, with a mean age of $30.42 \pm 7.67$ years. The following inclusion criteria were adopted: aged between 20 and 40 years, time greater than 12 months since injury, complete spinal cord injury ("A" classification in the scale of the American Spinal Injury Association - ASIA), stable clinical condition, nonsmoker, and not presenting acute respiratory complications or a history of cardiorespiratory diseases. The exclusion criteria considered were: spinal cord injury below $\mathrm{C} 8$ and inability to comprehend the protocol.

\section{Evaluation protocols}

All the volunteers underwent a preliminary evaluation which consisted of obtaining personal, demographic and anthropometric data.

\section{Pulmonary function}

In order to verify the presence of respiratory disturbances and thus characterize the population, the equations for the prediction of normal values, based on the equations for healthy subjects were used in accordance with the Guidelines for the pulmonary function tests (12). The pulmonary function tests were performed according to the guidelines of the American Thoracic Society - ATS (13) for technique, acceptability and reproducibility, using a spirometer (Easy One ${ }^{\mathrm{TM}}$, ndd Medizintechnik AG, Zurich, Switzerland). The system was calibrated before each test, according to the manufacturer's instructions. Spirometric variables were recorded and expressed in BTPS conditions (Body temperature and pressure saturated).

For the performance of the measurements, the subject rested for 10 minutes prior to the test and the procedures were carefully explained to him. The examination was performed with the volunteer sitting in his own wheelchair, with the back and seat fixed, providing a $90^{\circ}$ angle of hip flexion, the head was held in a neutral position and a nose clip used to prevent air leakage from the nostrils. The mouthpiece was attached to the mouth of the subject, avoiding any air leakage. The forced vital capacity (FVC) maneuver was performed until three acceptable and two reproducible curves were obtained. During its execution, real-time graphs of the curves were provided, indicating whether they met the acceptance criteria proposed by the ATS. The criteria were adjusted for subjects with spinal cord injury due to the muscle dysfunction present, with adjustments to the expiratory time and backextrapolated volume being required $(14,15)$. The FVC reference values were obtained from the spirometry test, forced expiratory volume in the first second $\left(\mathrm{FEV}_{1}\right)$ and the ratio $\mathrm{FEV}_{1} / \mathrm{FVC}$.

\section{Maximal inspiratory pressure}

MIP values were obtained using a previously calibrated digital respiratory pressure meter (model MVD300, GlobalMed, Porto Alegre, Brazil). The measurements were made with the subjects seated in their own wheelchairs, with their feet supported, and hips and knees at $90^{\circ}$. To measure MIP, the nostrils were occluded using a nasal clip. The measurement was performed during maximal inspiratory effort initiated from functional residual capacity (7). The volunteer breathed through a mouthpiece containing an adapter with a $2 \mathrm{~mm}$ opening. At least five, technically satisfactory, maximal inspiration efforts were carried out, i.e. without perioral air leakage, with inhalation maintained for at least $1 \mathrm{~s}$, and with values similar to each other $(\leq 10 \%)$, with the highest value considered for the study (16). 


\section{Sniff nasal inspiratory pressure}

The SNIP was measured with the volunteers in the same position. The measurement was made with one nostril occluded with a nasal silicone plug, which remained connected to the respiratory pressure meter by a catheter with an approximate diameter of $1 \mathrm{~mm}$ (17). The maneuver consisted of one maximum sniff performed through the contralateral (free) nostril, with the mouth closed, from functional residual capacity. Ten sniff test maneuvers were performed (18), with an interval of 30 seconds between each one, with the selection criterion used for an acceptable sniff being, a gradual pressure increase until reaching the peak, with a duration of up to 5 seconds (7). All the values were recorded on individual forms for each subject and the highest value was used for the data analysis. The volunteers were evaluated in a random order according to the methodologies used, and, at the end of the first examination, rested for 30 minutes prior to performing the subsequent measurement. The predicted MIP (16) and SNIP (7) values were based on equations for healthy subjects.

\section{Statistical analysis}

The values were expressed as mean and standard deviation, and the analysis of the data distribution was conducted using the Shapiro-Wilk test. Student's t-test was used to compare the values obtained from the two methodologies. To verify the relationship between the variables, Pearson's correlation coefficient was used, and for the analysis of concordance between the methodologies, the Bland-Altman method (19) was used. A statistical significance level of $\alpha=5 \%$ was adopted. The statistical procedures were performed using the GraphPad InStat version 3.05 and Medcalc version 11.5.0 applications.

\section{Results}

Table 1 presents the study sample characterization.

Table 2 shows the predicted and obtained MIP and SNIP values, in which no statistically significant differences can be observed when comparing the values obtained for the two study variables. In the comparison between the predicted and obtained values, regarding both MIP and SNIP, the obtained values were lower than the predicted values $(\mathrm{p}<0.05)$.

In the relation analysis between MIP and SNIP, the results showed positive, statistically significant correlation, and concordance between the values obtained from the two evaluation methods (Figure 1).

Table 1 - Characteristics of the volunteers studied $(n=17)$

\begin{tabular}{cc}
\hline Variables & Values \\
\hline Age (years) & $30.42 \pm 7.67$ \\
Height (cm) & $164.89 \pm 38.31$ \\
Body weight $(\mathrm{kg})$ & $81.78 \pm 34.22$ \\
BMI (kg/m²) & $22.30 \pm 3.63$ \\
Injury level & $\mathrm{C} 4-\mathrm{C} 7$ \\
Injury duration (months) & $84.36 \pm 49.13$ \\
$\mathrm{FVC}(\%)$ & $51.29 \pm 15.24$ \\
$\mathrm{FEV}_{1}(\%)$ & $52.52 \pm 21.28$ \\
$\mathrm{FEV}_{1} / \mathrm{FVC}$ & $100.82 \pm 19.70$ \\
\hline
\end{tabular}

Note: $\mathrm{BMI}=$ body mass index; $\mathrm{FVC}=$ forced vital capacity; $\mathrm{FEV}_{1}=$ forced expiratory volume in first second. Source: Research data. 
Table 2 - Maximal inspiratory pressure (MIP) and Sniff nasal inspiratory pressure (SNIP) predicted and obtained values

\begin{tabular}{ccc}
\hline & MIP $\left(\mathrm{cmH}_{2} \mathbf{0}\right)$ & SNIP $\left(\mathrm{cmH}_{2} \mathbf{0}\right)$ \\
\hline Predicted values & $128.92 \pm 7.18$ & $114.11 \pm 3.19$ \\
Obtained values & $88.42 \pm 29.39^{*}$ & $86.68 \pm 25.40^{*}$ \\
\hline
\end{tabular}

Note: ${ }^{*}=p<0.05$ : Predicted values vs. Obtained values.

Source: Research data.
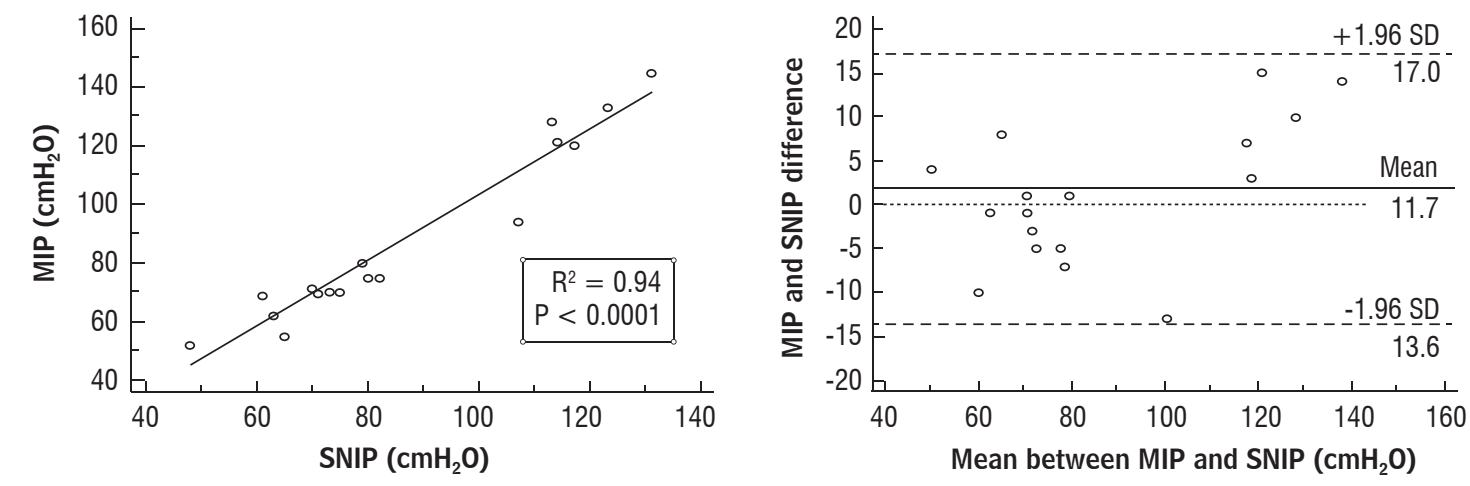

Figure 1 - Graphical representation of the correlation analysis between Maximal inspiratory pressure (MIP) and Sniff nasal inspiratory pressure (SNIP), in $\mathrm{cmH}_{2} \mathrm{O}$, and scatter plot for the difference and mean between and MIP and SNIP variables of the 17 tetraplegic volunteers studied

Source: Research data.

\section{Discussion}

In the present study, to better characterize the volunteers, the spirometric evaluation was performed at the beginning of the experimental protocol and the results indicated that the lung function of all participants was reduced compared to the predicted values. Changes in lung volumes and capacity are often caused by a muscle deficit (20), with the degree of compromise of these muscles dependant on the spinal cord injury level (21). The lack of coordination in the activation of the respiratory muscles, and the reduction in the volumes and capacities characterizes the non-parenchymal restrictive syndrome presented by this population (22).

The MIP and SNIP values obtained presented no difference when compared with each other, however, they were significantly lower compared to the predicted values, with the MIP values showing correlation and concordance with those of the SNIP. The maximal inspiratory pressure values below those predicted can be justified by the spinal cord injury, depending on the injury level, compromising the respiratory muscles, considering that the main inspiratory muscles have a higher location in the torso and are innervated by the upper spinal segments, thus the higher the level of the spinal cord injury, the greater the compromise to the inspiratory muscles (23). Another study evaluated the influence of the level of severity (partial or complete) and the duration of the spinal cord injury on the pulmonary function of quadriplegics and paraplegics, demonstrating that the higher, more severe, and longer duration of the injury, the worse the results obtained in the pulmonary function test, with the severity of the injury the factor of greatest importance in the reduction of pulmonary function (2).

Despite the difficulties mentioned in the literature regarding the measurement of MIP in people with spinal cord injuries, the significant correlation and concordance between the MIP and SNIP values obtained in this study are noteworthy since they 
indicate viability for the use of MIP for evaluating IMS in the study population, considering that in the presence of low MIP values, SNIP offers a way to differentiate inspiratory muscle weakness from difficulty in achieving sustained continual effort (7). There are publications on the use of SNIP in healthy subjects (7), neuromuscular disease patients (18, $24,25)$ and people with chronic obstructive pulmonary disease (26), however, studies with spinal cord injury patients are still scarce. Authors (27) observed that SNIP correlated positively and statistically significantly with the degree of spinal cord injury. They highlighted that SNIP proved to be better than MIP in discriminating the effect of the spinal cord injury level on IMS. From the preliminary results, the authors also concluded that SNIP is a more accurate measure of inspiratory function than MIP, which differs from the results of the present study, in which no difference between the values obtained through the two methods were found, however, the comparison between the two studies becomes unviable when considering the methodological differences between them.

In addition to SNIP allowing the differentiation between inspiratory muscle weakness and difficulties in performing the technique, it has other advantages, especially in individuals with tetraplegia, as SNIP consists of a simpler technique than that performed for the MIP measurement, with a lower risk of fatigue due to being a natural, easy and short maneuver, in which peak pressure needs to be sustained for a shorter duration (11). Furthermore, the determination of SNIP is considered a natural and easy procedure, which allows those evaluated to activate the inspiratory muscles with increased muscle fiber recruitment when compared to MIP, $100 \%$ and $61.1 \%$, respectively, according to a study that evaluated the amplitude of the peak electromyography activity of the diaphragm muscle during the performance of the two methods (28).

Considering that the procedures used to perform the MIP and SNIP measurements were not equivalent, it is worth highlighting that in the present investigation care was taken so that the measurements started from the same lung volume, i.e., functional residual capacity, thus avoiding the promotion of differences in the activation of inspiratory muscle patterns. Regarding the limitations of this study, the interpretation of the results presented should be taken into consideration, as predictive equations for healthy subjects were used, which may overestimate the difference between the predicted and obtained values. Another point to consider is the impossibility of performing a gold standard test (esophageal pressure) in order to compare the MIP and SNIP values obtained.

\section{Conclusion}

Both the MIP and SNIP values obtained proved lower than those predicted, indicating a reduction in IMS. However, despite being reported in the literature that MIP may not be an adequate technique for the evaluation of IMS in subjects with spinal cord injury, in the present study these values presented correlation and concordance with SNIP, which is referred to as a more accurate measure of inspiratory function in spinal cord injury patients, suggesting that MIP can be used as a noninvasive method for the evaluation of IMS in this population.

\section{References}

1. Hopman MT, Van der Woude LH, Dallmeijer AJ, Snoek G, Folgering HT. Respiratory muscle strength and endurance in individuals with tetraplegia. Spinal Cord. 1997;35(2):104-8.

2. Linn WS. Adkins RH, Gong H Jr., Waters RL. Pulmonary function in chronic spinal cord injury: a crosssectional survey of a large southern California outpatient population. Arch Phys Med Rehabil. 2000;81(6):757-63.

3. Mansel JK, Norman JR. Respiratory complications and management of spinal cord injuries. Chest. 1990; 97(6):1446-52.

4. Gounden P. Static respiratory pressures in patients with post-traumatic tetraplegia. Spinal Cord. 1997; 35(1):43-7.

5. Goldman JM, Rose LS, Williams SJ, Silver JR, Denison DM. Effect of abdominal binders on breathing in tetraplegic patients. Thorax. 1986;41(12):940-5.

6. Leith DE, Bradley M. Ventilatory muscle strength and endurance training. J Appl Physiol. 1976;41(4):508-16.

7. Uldry C, Fitting JW. Maximal values of sniff nasal inspiratory pressure in healthy subjects. Thorax. 1995; 50(4):371-5. 
8. Syabbalo N. Assessment of respiratory muscle function and strength. Postgrad Med J. 1998;74(870):208-15.

9. Souza RB. Pressões respiratórias estáticas máximas. J Pneumol. 2002;28(3):155-65.

10. Morgan RK, McNally S, Alexander M, Conroy R, Hardiman 0, Costello RW. Use of Sniff nasal-inspiratory force to predict survival in amyotrophic lateral sclerosis. Am J Respir Crit Care Med. 2005;171(3):269-74.

11. Prigent $H$, Lejaille $M$, Falaize L, Louis A, Ruquet M, Fauroux B, et al. Assessing inspiratory muscle strength by sniff nasal inspiratory pressure. Neurocritical Care. 2004;1(4):475-8.

12. Diretrizes para testes de função pulmonar. J Pneumol. 2002;28(Suppl 3):1-82.

13. American Thoracic Society. Standardization of spirometry 1994 update. Am J Respir Crit Care Med. 1995;152(3):1107-36.

14. Ashba J, Garshick E, Tun CG, Lieberman SL, Polakoff DF, Blanchard JD, et al. Spirometry-acceptability and reproducibility in spinal cord injured subjects. J Am Paraplegia Soc. 1993;16(4):197-203.

15. Kelley A, Garshick E, Gross ER, Lieberman SL, Tun CG, Brown R. Spirometry testing standards in spinal cord injury. Chest. 2003;123(3):725-30.

16. Neder JA, Andreoni S, Lerario MC, Nery LE. Reference values for lung function tests. II. Maximal respiratory pressures and voluntary ventilation. Braz J Med Biol Res. 1999;32(6):719-27.

17. Ruppel G. Lung volume tests. In: Mottram C. Manual of Pulmonary Function Testing. St Louis: Mosby; 1994. p. 1-25.

18. Lofaso F, Nicot F, Lejaille M, Falaize L, Louis A, Clement A, et al. Sniff nasal inspiratory pressure: with is the optimal number of sniffs? Eur Respir J. 2006;27(5):980-2.

19. Bland JM, Altman DG. Statistical methods for assessing agreement between two methods of clinical measurement. Lancet. 1986;1(8476):307-10.

20. Roth EJ, Lu A, Primack S, Oken J, Nusshaum S, Berkowitz $M$, et al. Ventilatory function in cervical and high thoracic spinal cord injury. Relationship to level of injury and tone. Am J Phys Med Rehabil. 1997; 76(4):262-7.
21. Winslow C, Rozovsky J. Effect of spinal cord injury on the respiratory system. Am J Phys Med Rehabil. 2003; 82(10):803-14.

22. De Troyer A. Respiration mechanics in tetraplegia. Bull Mem Acad R Med Belg. 1997;152(1):91-7.

23. Mueller G, Groot S, Woude LVD, Hopman MTE. Timecourses of lung function and respiratory muscle pressure generating capacity after spinal cord injury: a prospective cohort study. J Rehabil Med. 2008; 40(4):269-76.

24. Fitting JW, Paillex R, Hirt L, Aebischer P, Schluep M. Sniff nasal pressure: a sensitive respiratory test to assess progression of amyotrophic lateral sclerosis. Ann Neurol. 1999;46(6):887-93.

25. Stefanutti D, Benoist MR, Scheinmann P, Chaussain M, Fitting JW. Usefulness of sniff nasal pressure in patients with neuromuscular or skeletal disorders. Am J Respir Crit Care Med. 2000;162(4 Pt 1):1507-11.

26. Kyroussis D, Johnson L C, Hamnegard CH, Polkey MI, Moxham J. Inspiratory muscle maximum relaxation rate measured from submaximal sniff nasal pressure in patients with severe COPD. Thorax. 2002; 57(3):254-7.

27. Rocha AP, Mateus SRM, Horan TA, Beraldo PSS. Determinação não-invasiva da pressão inspiratória em pacientes com lesão medular traumática: qual é o melhor método? J Bras Pneumol. 2009;35(3):256-60.

28. Nava S, Ambrosino N, Crotti P, Fracchia C, Rampulla C. Recruitment of some respiratory muscles during three maximal inspiratory manoeuvres. Thorax. 1993; 48(7):702-7.

Received: 06/15/2013

Recebido: 15/06/2013

Approved: $12 / 23 / 2013$

Aprovado: 23/12/2013 